\title{
An outbreak of norovirus infection associated with fermented oyster consumption in South Korea, 2013
}

\author{
H. G. CHO ${ }^{1}$, S. G. LEE ${ }^{2}$, M. Y. LEE ${ }^{3}$, E. S. HUR ${ }^{1}$, J. S. LEE ${ }^{4}$, P. H. PARK ${ }^{1}$, \\ Y. B. PARK ${ }^{1}$, M. H. YOON ${ }^{1}$ AND S. Y. PAIK ${ }^{5 *}$ \\ ${ }^{1}$ Division of Public Health Research, Gyeonggi Province Institute of Health and Environment, Suwon, Republic of Korea \\ ${ }^{2}$ Korea Zoonosis Research Institute, Chonbuk National University, Iksan, Republic of Korea \\ ${ }^{3}$ An-Seong Public Health center, An-Seong, Republic of Korea \\ ${ }^{4}$ Food Microbiology Division, Food Safety Evaluation Department, National Institute of Food and Drug Safety \\ Evaluation, Osong, Republic of Korea \\ ${ }^{5}$ Department of Microbiology, College of Medicine, The Catholic University of Korea, Seoul, Republic of Korea
}

Received 31 August 2015; Final revision 16 November 2015; Accepted 14 January 2016;

first published online 2 February 2016

\section{SUMMARY}

An acute gastroenteritis (AGE) outbreak was reported in May 2013 in Gyeonggi Province, South Korea. Eight students who had eaten breakfast on 21 May 2013 at a high-school restaurant exhibited AGE symptoms. Our case-control study showed that a strong association was observed between AGE symptoms and fermented oyster consumption. Virological studies also indicated that noroviruses (NoVs) were detected from both clinical samples and fermented oyster samples, and multiple different genotypes (genogroups GII.4, GII.11 and GII.14) of NoVs were present in both samples. The nucleotide sequence similarity between the strains found in the clinical samples and those in the fermented oysters was more than $99 \cdot 5 \%$. Therefore, to prevent further outbreaks, proper management of raw oysters is necessary and the food industry should be aware of the risk of viral gastroenteritis posed by fermented oysters contaminated with NoVs.

Key words: Foodborne outbreak, gastroenteritis, norovirus, oysters.

\section{INTRODUCTION}

Noroviruses (NoVs) are now recognized as the leading aetiological agent of non-bacterial acute gastroenteritis (AGE) outbreaks, which are responsible for at least $50 \%$ of all AGE outbreaks worldwide, and a major cause of foodborne illness [1]. NoVs are extremely contagious and can infect people of all ages [1]. NoVs cause a self-limiting infection with AGE symptoms of typically 2-3 days duration. In the USA, NoVs cause 19-21 million illnesses and lead to 56000-70 000 hospitalizations and 570-800 deaths annually [2].

\footnotetext{
* Author for correspondence: Dr S. Y. Paik, Department of Microbiology, College of Medicine, The Catholic University of Korea, Seoul 137-701, Republic of Korea.

(Email: paik@catholic.ac.kr)
}

NoVs are transmitted via the faecal-oral route, and the infections usually spread through person-to-person contact, foodborne or waterborne [1]. Of these routes of transmission, the foodborne transmission of $\mathrm{NoV}$ infection typically occurs via an infected food handler and contaminated food items such as raw oysters, fruits, and vegetables [3]. Raw oysters are one of the well-known vehicles for the transmission of NoVs, which results in foodborne AGE outbreaks [4]. NoVs are known to be capable of persisting in the tissues of oysters for long periods [3]. Furthermore, oysters accumulate a large amount of NoVs when harvested from sewage-contaminated water [5]. Therefore, NoV outbreaks associated with raw oyster consumption have been well documented worldwide [3-5]. 
In South Korea, raw oysters are one of the favourite food items and are usually consumed during the winter season. Furthermore, the fermentation of raw oysters with $5-10 \%$ salt at room temperature for approximately 2 weeks, for the preparation of 'eoriguljeot', is a traditionally popular technique in South Korea practised so that raw oysters can be enjoyed over a longer period [6]. During 2007-2012, various seafood items including raw oysters were reported as a major vehicle and NoV was the most common pathogen identified in foodborne disease outbreaks in South Korea [7]. However, no epidemiological and virological studies of oyster-associated NoV outbreaks have been reported from South Korea.

In May 2013, an AGE outbreak was reported at a high school in Anseong, Gyeonggi Province, South Korea. We therefore conducted epidemiological investigations to characterize the extent of the outbreak, to identify the route of transmission, and to determine the causative agent with virological tests. Our findings indicate that the AGE outbreak was closely associated with NoVs from fermented oysters.

\section{METHODS}

\section{Epidemiological investigation}

On 24 May 2013, a physician reported a suspected AGE outbreak to a health centre after eight students from one particular high school visited the local clinic with typical AGE symptoms of diarrhoea, vomiting, abdominal pain and nausea. In accordance with the guidelines for epidemiological investigations for waterborne and foodborne diseases, published by the Korea Center for Disease Control \& Prevention (KCDC), all AGE outbreaks should be promptly and thoroughly investigated by technically trained public health officials to identify the causative agents and stop the spread of the disease $[8,9]$. Public health authorities visited the high school to investigate the outbreak and the eight affected students indeed showed AGE symptoms from 21 to 22 May 2013. The students were then asked to complete a standardized questionnaire to identify which food items from the school restaurant they consumed, along with clinical information about the AGE symptoms. The results of the questionnaire were used for a subsequent case-control study to detect the vehicle for this outbreak. Cases were defined as students who presented at least three episodes of diarrhoea; or at least one episode of vomiting, nausea, abdominal pain or fever within $72 \mathrm{~h}$ of eating breakfast at the school restaurant on 21 May 2013. Controls were defined as students who did not meet the definition of a case, but who ate breakfast at the school restaurant on 21 May. The number of control subjects was approximately three times that of the number of case subjects. Odds ratios (OR) with $95 \%$ confidence intervals (CI) and $P$ values to estimate the associations between the AGE symptoms and potential exposures were calculated using Excel 2010 (Microsoft Corp., USA).

\section{Collection of clinical, food, and environmental specimens}

On 24 May 2013, a total of 16 stool specimens were collected by public health officials from the eight AGE students and eight staff members who were working at the restaurant in the high school. The collected stool specimens were stored at $4{ }^{\circ} \mathrm{C}$ and were transferred to Gyeonggi Province Institute of Health and Environment (GIHE) in order to identify the aetiological agent responsible for the outbreak. In addition, samples of preserved food items served at breakfast on 21 May, together with environmental samples from kitchen knives, chopping boards, and dishcloths, and drinking-water and tap-water samples were collected and transferred to GIHE to identify the causative pathogens.

\section{Microbiological investigation}

The stool, food, and environmental samples collected were tested for ten species of bacteria (Escherichia coli, Salmonella spp., Shigella spp., Vibrio spp., Staphylococcus aureus, Clostridium perfringens, Campylobacter jejuni, Listeria monocytogenes, Yersinia enterocolitica, Bacillus cereus). The samples were cultured for bacterial pathogens at GIHE. Enteric bacterial pathogens were examined as described previously [9]. The stool samples and uncooked food samples (fermented oysters and cabbage kimchi) were tested for five species of viruses (norovirus, group A rotavirus, astrovirus, adenovirus, sapovirus) [9]. The drinking-water and tap-water samples from the school were tested for general bacteria, total coliforms, and E. coli [8].

\section{Norovirus detection and sequence analysis}

For virus detection, the faecal specimens were diluted to $10 \%$ in phosphate-buffered saline (PBS) and 
clarified by centrifugation at $800 \mathrm{~g}$ for $15 \mathrm{~min}$, and the supernatants were then collected. The fermented oyster samples were tested for the presence of norovirus by using proteinase $\mathrm{K}$ digestion [10]. Briefly, the fermented oyster samples were rinsed twice with water to remove the seasoning. The digestive diverticula were removed and dissected using sterile scissors and forceps. Approximately $1.5 \mathrm{~g}$ digestive tissues from the oysters was obtained and wholly homogenized with the same amount of proteinase $\mathrm{K}$ (SigmaAldrich, USA) and incubated at $37^{\circ} \mathrm{C}$ with shaking at $320 \mathrm{rpm}$ for $1 \mathrm{~h}$. The samples were then incubated at $60{ }^{\circ} \mathrm{C}$ for $15 \mathrm{~min}$. Next, the samples were clarified in two rounds of centrifugation at $4000 \mathrm{~g}$ for $5 \mathrm{~min}$, and the final supernatants were obtained for the measurements. Viral RNA was extracted from the $140 \mu 1$ supernatant of faecal and oyster samples using the QIAamp viral RNA mini kit (Qiagen, Germany) according to the manufacturer's instructions. To detect the NoVs, semi-nested reverse transcriptas-polymerase chain reaction (RT-PCR) was performed using specific primer sets (NV-GIF1M/NV-GIR1M/ NV-GIF2 for NoV GI; NV-GIIF1M/NV-GIIR1M/ NV-GIIF3M for NoV GII), targeting the capsid gene (region C) as described previously [8]. For human samples, the final PCR products were then purified and bi-directionally sequenced using nested PCR primers. For oyster samples, the amplified fragments were purified and then cloned into pGEM-T Easy vector (Promega, USA) according to the manufacturer's recommendations. Plasmids were purified and then sequenced. The MEGA software program v. 6.0 was used for the phylogenetic analysis with reference strains [11].

\section{RESULTS}

\section{Epidemiological analysis}

On 24 May 2013, a local clinic physician notified Anseong Public Health Centre that eight students from the same high school in Anseong, Gyeonggi Province, South Korea had developed AGE symptoms 2 days previously. Initial epidemiological investigations were undertaken to identify the common feature in the affected population. Two common factors were observed; first, all the patients used the dormitory of the school, and second, they all ate breakfast at the school restaurant. Students using the dormitory of the school usually eat breakfast at the school restaurant, whereas other students eat breakfast at home, which was one of the critical differences between the two sets of students. Furthermore, none of the students who did not use the dormitory showed AGE symptoms. Therefore, in order to identify the cause of this outbreak, epidemiological and microbial investigations focused on the 130 students using the dormitory and who ate breakfast at the school restaurant were conducted.

Of the 130 students, eight $(6 \cdot 2 \%)$ exhibited AGE symptoms (Table 1). The epidemic curve for this outbreak showed that the index case was found at 23:30 hours on 21 May, the other seven patients were identified by 22 May and no secondary cases were found. The main gastrointestinal symptoms reported were vomiting $(n=6)$, nausea $(n=6)$, diarrhoea $(n=5)$, fever $(n=5)$ and chills $(n=4)$ with a range of onset times from 16 to $40 \mathrm{~h}$ after eating breakfast at the school restaurant. The average incubation time was $26.9 \mathrm{~h}$ and the median duration of the illness was 2 days (Table 1).

A case-control study was performed using the asymptomatic students who used the dormitory of the school as control subjects. Although the OR of the fermented oyster samples was not calculated owing to zero count of not-eaten group in case subjects (Table 2), the results of our case-control study revealed that the AGE outbreak could be significantly linked to fermented oysters among the five food items served to the students (Table 2).

\section{Microbiological analysis}

NoVs were detected in the faecal samples of all eight AGE students, whereas none of the faecal samples from food handlers showed the presence of NoVs. None of the other enteric bacteria or viruses tested was found to be present in the same eight specimens. The drinking- and tap-water samples were also negative for general bacteria, total coliforms, and E. coli. Multiple genotypes of $\mathrm{NoV}$ were detected in the faecal specimens of the cases; NoV GII.4, GII.11 and GII.14 (Table 1, Fig. 1). Furthermore, the GII.4 and GII.14 NoV strains were also identified in fermented oysters served from the breakfast menu on the day. The nucleotide sequence similarity between the NoV strains from the clinical samples and those from the fermented oysters was $99 \cdot 5-100 \%$ (Fig. 1).

\section{DISCUSSION}

In this study, both the epidemiological and the virological evidence supported fermented oysters to be 
Table 1. Epidemiological features of the oyster-borne NoV-associated outbreak in Gyeonggi Province, South Korea, 24 May 2013

\begin{tabular}{|c|c|c|}
\hline \multicolumn{2}{|c|}{ Epidemiological feature } & Results \\
\hline \multicolumn{2}{|c|}{ Type of epidemiological study } & Case-control \\
\hline \multicolumn{2}{|c|}{ Outbreak setting } & High school \\
\hline \multicolumn{2}{|l|}{ Time of exposure } & 21 May 2013 (07:30) \\
\hline \multicolumn{2}{|l|}{ Date of onset } & $\begin{array}{l}21 \text { May } 2013(23: 30) \text { to } \\
22 \text { May } 2013(22: 00)\end{array}$ \\
\hline \multicolumn{2}{|c|}{ Attack rate (no. of ill cases/no. of cases exposed) } & $6 \cdot 2 \%(8 / 130)$ \\
\hline \multirow{5}{*}{\multicolumn{2}{|c|}{ Clinical symptoms }} & Diarrhoea (5) \\
\hline & & Chills (4) \\
\hline & & Fever (5) \\
\hline & & Nausea (6) \\
\hline & & Vomiting (6) \\
\hline \multicolumn{2}{|c|}{ Mean incubation time (range) } & $26 \cdot 9(16-40) \mathrm{h}$ \\
\hline \multicolumn{2}{|c|}{ Median duration of illness (range) } & $2(1-2)$ days \\
\hline \multicolumn{2}{|c|}{ Mean age } & 17 years \\
\hline \multicolumn{2}{|l|}{ Route of exposure } & Fermented oysters \\
\hline \multirow[t]{3}{*}{ Clinical specimens } & Total & 8 \\
\hline & NoV-positive & 8 \\
\hline & Genotype $(n)$ & GII.4 (2), GII.11 (2), GII.14 (4) \\
\hline \multirow[t]{4}{*}{ Fermented oysters } & Collection points & School restaurant \\
\hline & NoV genotype & GII.4, GII.14 \\
\hline & Homology* & $>99 \cdot 5 \%$ \\
\hline & Other viruses $\dagger$ & Negative \\
\hline
\end{tabular}

* Compared to linked clinical specimens.

$\dagger$ Other viruses included group A rotavirus, enteric adenovirus, astrovirus, and sapovirus.

Table 2. Association between the acute gastroenteritis symptoms and the breakfast menu served on 21 May 2013 in the school restaurant

\begin{tabular}{|c|c|c|c|c|c|c|}
\hline \multirow[b]{2}{*}{ Food items } & \multicolumn{2}{|c|}{ Cases $(n=8)$} & \multicolumn{2}{|c|}{ Controls $(n=27)$} & \multirow[b]{2}{*}{ OR $(95 \% \mathrm{CI})$} & \multirow[b]{2}{*}{$P$ value } \\
\hline & Ate & Did not eat & Ate & Did not eat & & \\
\hline Beef soup & 7 & 1 & 15 & 9 & $4.20(0.44-39.94)$ & $0 \cdot 3784$ \\
\hline Fermented oyster & 8 & 0 & 9 & 18 & - & 0.0036 \\
\hline Hamburger steak & 7 & 1 & 22 & 4 & $1.27(0 \cdot 12-13 \cdot 35)$ & 0.7119 \\
\hline Scrambled egg & 7 & 1 & 23 & 3 & $0.91(0.08-10 \cdot 23)$ & 0.5798 \\
\hline Cabbage kimchi & 4 & 3 & 14 & 11 & $1 \cdot 05(0 \cdot 19-5 \cdot 70)$ & 0.7061 \\
\hline
\end{tabular}

OR, Odds ratio; CI, confidence interval.

associated with the NoV outbreak that occurred at a high school in May 2013 in South Korea. The affected students suffered from AGE symptoms, and analysis of the faecal specimens obtained from the symptomatic students confirmed NoVs as the causative agent. The results of case-control study suggested a strong correlation between AGE symptoms and consumption of fermented oysters. NoVs were detected in fermented oysters and the sequence similarity between the NoVs from the clinical and fermented oyster samples was found to be more than $99 \cdot 5 \%$.
The association between AGE outbreaks and the consumption of shellfish is well established [3]. From 2006 to 2013, $102 \mathrm{NoV}$ outbreaks were reported in Gyeonggi Province, South Korea [12]. Fifteen of these outbreaks were associated with bivalve shellfish such as oysters and mussels, but no NoVs were detected in the shellfish owing to the lack of leftover samples, although groundwater and kimchi were identified as vehicles in the NoV outbreaks [8]. However, fermented oysters were fortunately obtained as preserved food by the school in this outbreak, and 


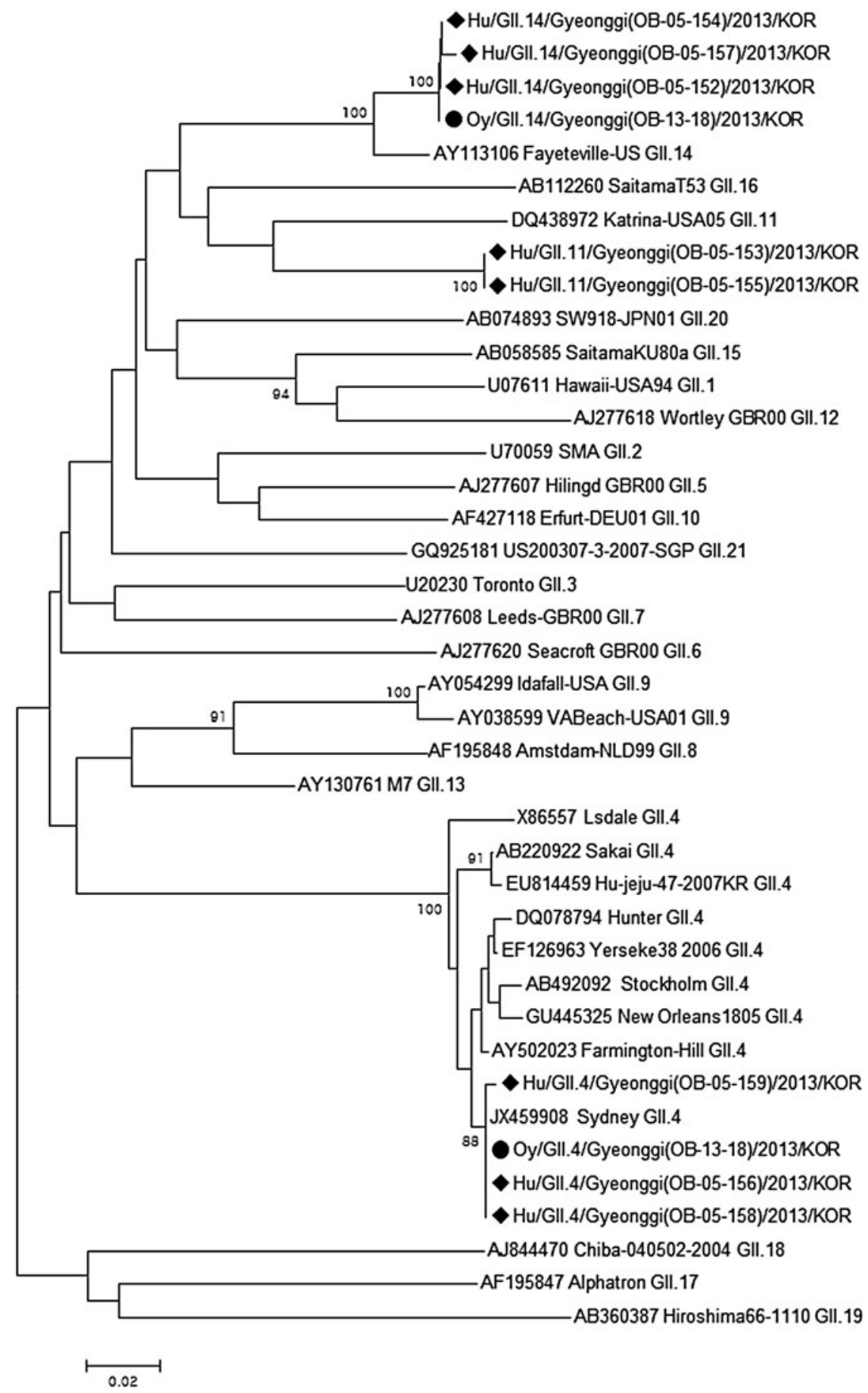

Fig. 1. A phylogenetic tree was constructed using 218 base-pair nucleotide sequences from the capsid gene of the NoV strains isolated from the clinical specimens $(\bullet)$ and fermented oyster samples $(\bullet)$ associated with the acute gastroenteritis outbreak. ClustalW and the neighbour-joining method with bootstrap analysis $(n=1000)$ were used for DNA sequence alignments and dendrogram construction, respectively. Oy, Fermented oyster; Hu, human patient; OB, outbreak.

multiple NoV genotypes were identified from these fermented oysters, which was consistent with other previous reports of oyster-borne NoV outbreaks $[3,5,10]$.

Fermented oysters are one of the representative fermented foods and have never been reported as a vehicle of transmission in NoV outbreaks in South Korea previously [6]. A recent study reported that murine and feline NoVs were significantly inactivated in fermented oysters during the 2 weeks of fermentation [6]. However, in the present study, fermented 
oyster samples could not be obtained directly from the food company that supplied them to the school. Furthermore, this company was not the manufacturer, only a mid-distributor in the complex distribution system of fermented oysters. Therefore, additional information about the fermented oysters could not be obtained because the company did not know the production area or the fermentation period of these oysters. Moreover, since none of the faecal samples from the food handlers at the high school showed the presence of NoVs, contamination of the food by asymptomatic NoV carriers at the high school is unlikely.

Only eight of the 130 exposed students were affected. This low attack rate can be explained by individual preference for fermented oysters; fermented oysters develop a distinctive flavour after curing, therefore they may not be preferred by many of the students [6]. In addition, a dose-dependent relationship between fermented oyster consumption and AGE symptoms is considered as a strong indicator of the involvement of the oysters in AGE outbreaks [13]. However, this aspect was not studied in this epidemiological investigation, which was one limitation of this study. Despite the limitations mentioned above, based on our results, we assume that raw oysters contaminated by NoVs were used to prepare the fermented oysters, and that these fermented oysters harbouring active NoVs were served to the students, which resulted in this AGE outbreak.

In conclusion, our results indicate that the fermented oysters were very likely to be the vehicles for NoVs responsible for this AGE outbreak. Our findings can contribute to a greater understanding of the risks associated with the consumption of NoV-contaminated oysters. Therefore, to prevent further outbreaks, proper management of raw oysters prior to the preparation of fermented oysters is necessary, and the food industry should be aware of the risks of viral gastroenteritis posed by the consumption of fermented oysters contaminated with NoVs.

\section{ACKNOWLEDGMENTS}

This study was supported by the Korea Environmental Industry \& Technology Institute (2013000550009) and a grant of the Korea Health Technology R\&D Project through the Korea Health Industry Development Institute (KHIDI), funded by the Ministry of
Health \& Welfare, Republic of Korea (grant no.: HI15C1781).

\section{DECLARATION OF INTEREST}

None.

\section{REFERENCES}

1. Hall AJ, et al. Updated norovirus outbreak management and disease prevention guidelines. Morbidity and Mortality Weekly Report. Recommemdations and Reports 2011; 60: 1-18.

2. Hall AJ, et al. Norovirus disease in the United States. Emerging Infectious Diseases 2013; 19: 1198-1205.

3. Le Guyader FS, Atmar RL, Le Pendu J. Transmission of viruses through shellfish: when specific ligands come into play. Current Opinion in Virology 2012; 2: 103-110.

4. Costantini V, et al. Human and animal enteric caliciviruses in oysters from different coastal regions of the United States. Applied and Environmental Microbiology 2006; 72: 1800-1809.

5. Le Guyader FS, et al. Detection of multiple noroviruses associated with an international gastroenteritis outbreak linked to oyster consumption. Journal of Clinical Microbiology 2006; 44: 3878-3882.

6. Seo DJ, et al. Inactivation of murine norovirus and feline calicivirus during oyster fermentation. Food Microbiology 2014; 44: 81-86.

7. Moon S, et al. Emerging pathogens and vehicles of food- and water-borne disease outbreaks in Korea, 2007-2012. Osong Public Health and Research Perspectives 2014; 5: 34-39.

8. Cho HG, et al. Acute gastroenteritis outbreaks associated with ground-waterborne norovirus in South Korea during 2008-2012. Epidemiology and Infection 2014; 142: 2604-2609.

9. Park JH, et al. Three gastroenteritis outbreaks in South Korea caused by the consumption of kimchi tainted by norovirus GI.4. Foodborne Pathogens and Disease 2015; 12: $221-227$.

10. Rajko-Nenow $\mathbf{P}$, et al. Norovirus genotypes present in oysters and in effluent from a wastewater treatment plant during the seasonal peak of infections in Ireland in 2010. Applied and Environmental Microbiology 2013; 79: 2578-2587.

11. Jeong AY, et al. Occurrence of norovirus infections in asymptomatic food handlers in South Korea. Journal of Clinical Microbiology 2013; 51: 598-600.

12. Cho HG, et al. Emergence of Norovirus GII.4 variants in acute gastroenteritis outbreaks in South Korea between 2006 and 2013. Journal of Clinical Virology 2015: 72: 11-15.

13. Loury $\mathbf{P}$, et al. A norovirus oyster-related outbreak in a nursing home in France, January 2012. Epidemiology and Infection 2015; 143: 2486-2493. 